\title{
Elimination of Chrysanthemum stunt viroid (CSVd) from an Viroidinfected Chrysanthemum through Shoot Tip Culture
}

\author{
Su-Min Jeon1, Wina Dian Savitri1, Kyeung-II Park1, Mi-Young Chung2, and Chang-Kil Kim1* \\ 1Department of Horticultural Science, Kyungpook National University, Daegu 702-701, Republic of Korea \\ 2Department of Agricultural Education, Sunchon National University, Sunchon 540-742, Korea \\ ( ${ }^{*}$ Corresponding author: ckkim@knu.ac.kr)
}

\begin{abstract}
As the increase of chrysanthemum demand on chrysanthemum increases in Korea, the production of high quality chrysanthemum is needed. Chrysanthemum stunt viroid (CSVd) is one of the important viroid, which infects chrysanthemum and induces diseases that affects the decrease of quality and yield. To solve this problem, we used different size of meristem of chrysanthemum 'Ency' for shoot tip culture and also that of combined with heat treatment at 37oC. The efficiency of CSVd elimination was influenced by the size of shoot tip. The small-sized of meristems with 1 or 2 leaf primodia were regenerated into the highest number of CSVd-free plantlets. By RT-PCR, the 214-bp band corresponding to CSVd was not detected in $22.2 \%$ of the total number of tested regenerants from shoot tips with 2 leaf primordia. While, shoot tip culture combined with heat treatment of one-month-old in vitro shoots was not effective for CSVd-elimination. The CSVd-free plants grew more vigorously than CSVd-infected plants in the greenhouse.
\end{abstract}

